\title{
Supine Versus Prone Percutaneous Nephrolithotomy - A Randomised Comparative Study
}

\author{
Mohan Keshavamurthy, Niramya Pathak, Karthik Rao", Sreeharsha Harinatha, Shakir Tabrez, \\ Premakumar Krishnappa, Basavaraja Neelagar, Santosh Kumar Subudhi
}

Department of Urology, Fortis Hospital, Bengaluru, India

\section{Email address:}

minorcalyx@gmail.com (M. Keshavamurthy),niramya.pathak@gmail.com (N. Pathak), bkarthikrao@gmail.com (K. Rao), drsreeharsh@gmail.com (S. Harinatha), docshakir@gmail.com (S. Tabrez), drpremkumark@gmail.com (P. Krishnappa), kbr124@gmail.com (B. Neelagar), subusan78@gmail.com (S. K. Subudhi)

${ }^{*}$ Corresponding author

\section{To cite this article:}

Mohan Keshavamurthy, Niramya Pathak, Karthik Rao, Sreeharsha Harinatha, Shakir Tabrez, Premakumar Krishnappa, Basavaraja Neelagar, Santosh Kumar Subudhi. Supine Versus Prone Percutaneous Nephrolithotomy - A Randomised Comparative Study. International Journal of Clinical Urology. Vol. 5, No. 1, 2021, pp. 51-57. doi: 10.11648/j.ijcu.20210501.21

Received: April 22, 2021; Accepted: May 31, 2021; Published: June 9, 2021

\begin{abstract}
Percutaneous Nephrolithotomy (PCNL) is the standard procedure for large renal stones. This study was conducted to compare the conventional Prone position PCNL with the newer concept of Supine PCNL. A prospective, randomised controlled, double blind study was conducted in 100 patients planned for PCNL. They were randomised into 2 groups with 50 patients each and PCNL was performed either in the prone or supine position. The patient groups were compared for the length of hospital stay, duration of surgery, postoperative and intra operative complications, postoperative stone free status, and requirement of adjunctive procedures. Stone free rates were significantly better for the supine PCNL group. Post operative complications such as fever was more for Prone PCNL group. The other parameters that were not statistically significant were mean operating time which was less for the supine group and duration of hospital stay which was less for the supine group. There was no difference in the other complication rates between the two procedures. The requirement of additional procedures for stone clearance were also same between both the groups. To conclude, Our study demonstrates that supine PCNL is a better technique than prone PCNL in terms of stone free rates, post-operative complications such as fever, lesser number of punctures required for stone clearance and more tubeless procedures.
\end{abstract}

Keywords: Supine PCNL, Prone PCNL, Post Operative Complications

\section{Introduction}

Percutaneous Nephrolithotomy (PCNL) is the gold standard procedure and treatment of choice for the treatment of large stones $(>2 \mathrm{~cm})$ and complex renal stones. [1, 2]

Simple puncture of the kidney from the flank was performed by Hillier in 1865 [3]. It was brought into widespread use by Kuster in 1865 [3]. J Israel and W. Israel mentioned percutaneous nephrolithotomy drainage in 1925 in their German textbook " Chirurgie der Niereund des Harnleiters' which was done using trocar puncture of hydronephrotic kidneys from the flank and they quoted Scheele to have performed this procedure around 1880 [3]. In 1941, Rupel and Brown described the first extraction of a stone using a cystoscope via a nephrostomy channel and following this, the first pyeloscope was developed by Trattner in 1948 [4]. In 1955, accidentally, Willard Goodwin, punctured the dilated renal pelvis with the patient in prone position for a translumbar aortogram. Goodwin, later expanded on the 'procedural mishap' and developed the technique of percutaneous pyelostomy in the prone position [4].

Percutaneous Nephrolithotomy was not widely accepted until the 1970s [3]. Percutaneous Nephrolithotomy under Ultrasound guidance was performed by Peddersen in 1974 [3]. Till that time, the procedure was relatively disregarded and was mainly in the hands of radiologists. But with the introduction of ultrasound guidance, the procedure was taken over by Urologists [3]. The first complete percutaneous 
Nephrolithotomy was described by Fernstrom and Johansson in 1976 and was performed in the prone position [5]. Expanding on this, Arthur Smith in 1978 described the first antegrade stent placement through a nephrostomy tract [4]. Subsequently, in collaboration with Drs Zuniga, Clayman and Amplatz, early articles were published on a series of 63 calculi extracted from 25 patients with a high success rate [4]. Between 1976 and 1979 Alken P, the radiologist Rolf Gunther and the Urologist Gerd Hutschenreiter contributed to further development of PNL technique [3]. Smith et al in 1979 coined the term Endourology [3]. After this period, the use of PNL expanded rapidly. Clayman and coworkers were the first to describe use of ballon dilation catheters for tract dilation in 1983. Since 1980, Marberger and collaborators designed a purposely built nephroscope and ultrasound lithotrite for percutaneous use together with the Richard Wolf $\mathrm{GmbH}$, Knittlingen, Germany, and Korth with Olympus Winter und Ibe, Hamburg, Germany. Also, Clayman and Castaneda-Zuniga were the first to publish a book on almost every aspect of percutaneous renal surgery [3]. Also the Amplatz dilators and sheath became widespread access instruments. Segura and coworkers were the first to publish a series of 1,000 procedures. Many other Urologists have contributed to this technique and they, like Clayman and collaborators in 1984, reported in the early 1980s that PNL had replaced $90 \%$ or more of their surgical procedures for renal stone removal [3].

Prone PCNL involves posterior renal access through Brodel's avascular line. Ureteric catheter insertion is initially undertaken cystoscopically with the patient placed in the lithotomy position, and then retrograde pyelography can be performed. The patient is then moved into the prone position. Supine PCNL involves positioning the patient supine with the side of interest on the lateral extreme of the operating table. The ipsilateral flank is elevated with a 1-3 $\mathrm{L}$ bag of fluid placed under the lumbar fossa on the operating side. The ipsilateral arm is positioned across the thorax and soft pads applied to pressure points. In the supine position, retrograde placement of the ureteric catheter, to obtain a retrograde pyelography and aid renal access puncture, is acquired through the easily accessible urethral meatus.

Prone patient position was chosen intuitively, based on anatomical considerations related to the posterior retroperitoneal location of the kidneys, the short access to posterior calyces situated on the avascular line of Brodel and the large surface area for puncture [6]. Prone PCNL became widely popular and totally replaced open renal stones surgery, emerging as the standard operation and exclusive position for 2 decades. Aiming to reduce patient, anaesthesia and surgery related inconveniences of the prone position, Valdivia et al. [7] described the performance of PCNL with the patient in the supine position in 1987. A recent study by the Endourological Society found that $80.3 \%$ of patients were operated in the prone position compared with $19.7 \%$ in the supine position [8].

Various modifications of supine pcnl are described with the purpose to ease access to urethra for simultaneous retrograde intra renal surgery while facilitating calyceal puncture and increasing flank exposure. The original supine position described by Valdivia did not gain in popularity for many years until Ibarluzea et al improvised it further by adding a modified lithotomy arrangement giving origin to the Galdakao-modified supine Valdivia (GMSV) position [9]. This position is widely used because it allowed simultaneous retrograde access. Two urologists from Orbassano (Torino), Dr. Roberto Mario Scarpa and Dr. Cesare Marco Scoffone, enthusiastic about the simultaneous endourological access, were the ones who created the acronym ECIRS (Endoscopic Combined IntraRenal Surgery). This position is used in the present article wherever supine PCNL is described.

\section{Patients and Methods}

Aim was to compare between Supine and Prone PCNL at Fortis Hospitals, Bannerghatta Road, Bangalore. Primary objectives were to compare Stone Free Rates (SFR) $(<4 \mathrm{~mm})$ and Mean operating times and Secondary objectives were to compare immediate and intermediate complications, Duration of stay in hospital, Requirement of Second stage PCNL or other ancillary procedures.

Patients posted for percutaneous nephrolithotomy, following approval by the institutional ethical committee of our institute from July 2017 to April 2019 were included as a randomised, double blinded, single centre, prospective, experimental comparative study. Patients with Renal stones ( $>1 \mathrm{~cm}$ size), Upper ureteral stones $(>1 \mathrm{~cm}$ size), Patients of all age groups and of both sexes were included in the study. Patients with bleeding disorders, pregnancy, active UTI, previous nephrostomy, patients not consenting for the procedure were excluded from the study. Randomisation is done by computer generated sequence. History and Clinical assessment was done. CT Urography with $3 \mathrm{D}$ reconstruction scan was done preoperatively and plain CT KUB was done postoperatively. 100 cases, 50 cases each of supine and prone PCNL, with above mentioned inclusion and exclusion criteria were included in the study.

Data was entered into data analysis software. Results on continuous measurements are presented on Mean \pm SD (MinMax) and results on categorical measurements are presented in Number (\%). Significance is assessed at 5\% level of significance. Student $t$ test (two tailed, independent) has been used to find the significance of study parameters on continuous scale between two groups (Inter group analysis) on metric parameters. Chi-square/ Fisher Exact probability test has been used to find the significance of study parameters on categorical scale between two or more groups. Approval by the ethics committee and scientific committee was obtained beforehand. All the patients fulfilling the selection criteria were explained regarding the nature of the study. Written informed consent taken in English and local language before enrolment. 


\section{Results}

\subsection{Patient and Preoperative Characteristics}

Table 1 outlines the patient characteristics of supine and prone PCNL patients. Supine PCNL had lesser proportion of male patients but the difference was not statistically significant $(56 \%$ vs $64 \%, p=0.25)$. Mean age was lesser in supine PCNL but difference was not statistically significant (45.7 vs 47.7, $\mathrm{p}=0.5$ ). Mean BMI was more in supine PCNL with no significant difference (24.6 vs $24, \mathrm{p}=0.49$ ). Mean Duration of hospital stay was lesser in supine PCNL with no significant difference $(2.84$ vs $2.98, \mathrm{p}=0.6)$. Mean stone diameter in supine PCNL was lesser with a significant difference $(2.85$ vs $3.65, \mathrm{p}=0.01)$. Single stones were present more in supine group with ( $78 \%$ vs $70 \%, p=0.2)$. Mean Guys stone score was less in Supine group (1.9 vs 2.1, $\mathrm{p}=0.4$ ). Anatomic abnormality was more in supine group (4\% vs $2 \%$, $\mathrm{p}=0.4)$. Simultaneous ureteral stone were present in equal proportions in both groups $(12 \%)$.

\subsection{Intraoperative Characteristics}

Table 1 outlines the intraoperative parameters of supine and prone PCNL patients. Supine group had more tubeless procedures $(46 \%$ vs $20 \%, \mathrm{p}=0.003)$ and a shorter mean duration of surgery (101.74 vs 102 min., $p=0.9$ ). Number of punctures were lesser in supine group ( 1 vs $1.24, \mathrm{p}=0.002$ ). Radiation exposure duration was more in supine PCNL group with a no significant difference ( 5.09 vs $4.78, p=0.79)$. Fall in hematocrit was more in supine group which was not statistically significant (5.56 vss $5.25, \mathrm{p}=0.68$ ). Requirement of blood transfusion was more in supine group than prone group ( $14 \%$ vs $12 \%, p=0.67)$. Intraoperative complications in form of perforation of PCS was seen in 1 patient in each study group. Supracostal approach was chosen more for supine group ( $2 \%$ vs $0, \mathrm{p}=0.16)$.

\subsection{Postoperative Parameters}

Supine PCNL group had a greater percentage of stone clearance which was significant $(76 \%$ vs $60 \%, \mathrm{p}=0.019)$. Requirement of second stage PCNL or ancillary procedures was present in $8 \%$ of both groups. Post operative fever was significantly less in supine group ( $0 \%$ vs $8 \%, p=0.005)$. Mean pain score was less for supine group which was significant (4.04 vs 4.72, $\mathrm{p}=0.04)$. Clavien Dindo complications grade 1 were seen in $25 \%$ and $22 \%$ of supine and prone groups and grade 2 were seen in $3 \%$ and $4 \%$ of supine and prone groups and the difference was not statistically significant. Catheter removal, stent removal and PCN tube removal day were comparable between the two groups

Table 1. Supine vs Prone PCNL.

\begin{tabular}{|c|c|c|c|}
\hline Patient Characteristics & Supine & Prone & P Value \\
\hline SAMPLE SIZE (n) & $\mathrm{n}=50$ & $\mathrm{n}=50$ & \\
\hline Gender (\% Male / \% Female) & $56 \% / 44 \%$ & $64 \% / 36 \%$ & 0.2538 \\
\hline Age in Years (Mean/SD) & $45.72(15.3)$ & $47.72(14.28)$ & 0.5024 \\
\hline BMI (Mean / SD) & $24.6(5)$ & $24(3.55)$ & 0.4923 \\
\hline Duration of stay & $2.84(1.45)$ & $2.98(1.96)$ & 0.6865 \\
\hline \multicolumn{4}{|l|}{ INVESTIGATIONS - CT SCAN } \\
\hline Mean stone diameter (SD) & $2.85(1.22)$ & $3.65(1.78)$ & 0.0116 \\
\hline Min / Max Stone Diameter & $1 / 3.4$ & $1.1 / 7.02$ & \\
\hline \multicolumn{4}{|l|}{ Number of Stones } \\
\hline Single (Number with Percentage) & $39(78 \%)$ & $35(70 \%)$ & 0.2032 \\
\hline Multiple (Number with Percentage) & $11(22 \%)$ & $15(30 \%)$ & 0.2032 \\
\hline Guys stone score (Mean/SD) & $1.9 / 1.3$ & $2.1 / 1.23$ & 0.4332 \\
\hline Anatomic abnormality & $2(4 \%)$ & $1(2 \%)$ & 0.4111 \\
\hline Simultaneous ureteral stone & $6(12 \%)$ & $6(12 \%)$ & 1.0000 \\
\hline \multicolumn{4}{|l|}{ Intraoperative parameters } \\
\hline \multicolumn{4}{|l|}{ a. Tube requirement } \\
\hline i. Tube (Number with Percentage) & $27(54 \%)$ & $40(80 \%)$ & 0.0003 \\
\hline ii. Tubeless (Number with Percentage) & $23(46 \%)$ & $10(20 \%)$ & 0.0003 \\
\hline b. Duration of surgery (Mean /SD) & $101.74(54.38)$ & $102(35.10)$ & 0.9775 \\
\hline c. Number of punctures (Mean / SD) & $1(0)$ & $1.24(0.52)$ & 0.0020 \\
\hline d. Radiation exposure duration (Mean / SD) & $5.09(1.89)$ & $4.78(2.02)$ & 0.4319 \\
\hline e. Fall in hematocrit (Mean / SD) & $5.566(3.74)$ & $5.254(3.84)$ & 0.6831 \\
\hline f. Requirement of blood transfusion & $7(14 \%)$ & $6(12 \%)$ & 0.6759 \\
\hline g. Intraoperative complications & $1(2 \%)$ & $1(2 \%)$ & 1.0000 \\
\hline \multicolumn{4}{|l|}{ h. Approach } \\
\hline i. Supracostal - number and percentage & $1(2 \%)$ & $0(0 \%)$ & 0.1616 \\
\hline ii. Subcostal - number and percentage & $49(98 \%)$ & $50(100 \%)$ & 0.1616 \\
\hline \multicolumn{4}{|l|}{ Post operative parameters } \\
\hline a. Stone clearance (percentage) & $38(76 \%)$ & $30(60 \%)$ & 0.0190 \\
\hline $\begin{array}{l}\text { b. Requirement of second stage penl/ancillary procedures } \\
\text { c. Complications }\end{array}$ & $4(8 \%)$ & $4(8 \%)$ & 1.0000 \\
\hline i. Clavien Dindo grade (Mean/SD) & $1.28(0.73)$ & $1.28(0.67)$ & 1.0000 \\
\hline CDG 1 & $42(84 \%)$ & $41(82 \%)$ & \\
\hline CDG 2 & $4(8 \%)$ & $5(10 \%)$ & \\
\hline
\end{tabular}




\begin{tabular}{llll}
\hline Patient Characteristics & Supine & Prone & P Value \\
\hline CDG 3 & $2(4 \%)$ & $3(6 \%)$ & \\
CDG 4 & $2(4 \%)$ & $1(2 \%)$ & 0.0058 \\
CDG 5 & $0(0 \%)$ & $0(0 \%)$ & 0.6759 \\
ii. Fever & $0(0 \%)$ & $6(8 \%)$ & 0.0413 \\
iii. Blood transfusion requirement & $7(14 \%)$ & $4.72(1.64)$ & 0.5678 \\
iv. Pain score (Mean /SD) & $4.04(1.6)$ & $2.16(1.15)$ & 0.7890 \\
d. Catheter removal day (Mean/SD) & $2.04(0.92)$ & $2.3414(1.216)$ & 0.2164 \\
e. Tube removal day (Mean/SD) & $2.2758(1.22)$ & $17.96(4.38)$ & \\
f. Stent removal day (Mean / SD) & $16.76(5.16)$ & & \\
\hline
\end{tabular}

\begin{tabular}{llllll}
\hline \multicolumn{2}{l}{ Clavien Score and Patient Position } & & & & Total \\
\hline \multirow{2}{*}{ Clavien Score } & Supine & & Prone & \% Age & No of patients \\
\cline { 2 - 6 } & No of Patients & \% age & No of patients & $48 \%$ & 46 \\
\hline NONE & 22 & $44 \%$ & 24 & $44 \%$ & 47 \\
I & 25 & $50 \%$ & 22 & $8 \%$ & 7 \\
II & 3 & $6 \%$ & 4 & $100 \%$ & 100 \\
Total & 50 & $100 \%$ & 50 & & \\
\hline
\end{tabular}

\section{Discussion}

Advantages of prone PCNL include: Posterior retroperitoneal location of kidney requiring short access to posterior calyces along the avascular line; Large surface area for puncture; Reduced risk of interposition of other viscera along the working tract; Surgeon experience [6]; Beneficial results in obese patients and staghorn calculus [10].

Disadvantages of prone position include: It compromises blood circulation and ventilation, especially in obese patients (limitation in respiratory movement); Position changes during the procedure is inevitable, because pre-placement of a ureteral catheter is commonly required in the dorsal lithotomy position before turning the patient to the prone position. These prolong the duration of the procedure. If the procedure is carried out under spinal or epidural anaesthesia, conversion to general anaesthesia with endotracheal intubation will represent a great challenge to the anaesthetist; Sometimes it is impossible for the patient to lie prone because of body habitus such as ankylosing spondylitis, severe lardosis or kyphosis, or hip or lower limb contractures Operating on a patient in the prone position, the surgical team stands in close proximity to the patient, making them relatively more vulnerable to radiation exposure. Whereas in the supine position, the bodies and limbs of the surgical team remain outside the field of the fluoroscope. The prone position is especially dangerous in patients with severe cervical spondylosis, and care of the pressure area is problematic [11].

Advantages of supine PCNL include: patient position needn't be changed; Ability to perform simultaneous ureteroscopy during PCNL; Easier air way control by the anaesthesiologist; Easier clearance of upper calyceal stones; Evacuation of stone fragments; Decreasing operating time; No contact between the patient's skin and water, which would prevent hypothermia; Less kidney displacement; Dependent Amplatz sheath drainage facilitating the spontaneous evacuation of stone fragments; Less retrorenal colon injury; More comfortable for corpulent or obese patients and patients with respiratory or cardiac problems.

There are numerous advantages for the endourologists in performing PCNL in the supine position such as: The fluoroscopy tube is far from the working space, reducing the radiation exposure for surgeon; Lack of overlapping of the vertebrae with the Kidney; Decreasing the total operating time; Sitting position for the surgeon; Supine PCNL is safe, effective, and suitable for most of the patients, and is feasible for all types of stones, such as calyceal, pelvic, multiple, staghorn, or upper pole calyceal stones (10), (12).

Disadvantages of supine PCNL include: There is not enough space for a third tract if needed; Access to the anterior and upper calyxes is more difficult (as the angle between the plane of the operation table and the anterior calyxes is less than that in other positions), it is difficult to access the calculi in the anterior calyxes; Approaching the upper calyx, especially if placed excessively medially is more difficult in supine position, as well as more chances of bleeding in access through lower calyx; The mobility of kidneys is more than that in the prone position enabling the kidneys to move anteromedially during tract dilation in the supine position; Finally, the pelvicaliceal system is constantly collapsed in this position, and consequently, nephroscopy is more difficult. [10, 11].

In order to establish advantages and disadvantages of both supine and prone position and to decide which method is more efficient and possibly safer, more comparative studies are necessary. Unfortunately, the results currently reported by different centres are not standardised. Also in different series, the complexity of the cases may be very dissimilar. Only recently Thomas and colleagues have recommended a novel scoring system [13]. Guy's stone score takes into account the complexity of the stone burden as well as the patient's anatomy, in order to predict preoperatively the likelihood of a stone-free outcome. So, in order to improve the heterogeneity of reporting outcomes and to bring a better correlation between the theoretical and evidence based literature reporting of the important parameters of prone and supine PCNL, we chose to do a prospective Randomised controlled comparative study between supine and prone PCNL. 
Demographic data:

Demographic data comparing Age, Sex, BMI, showed no statistically significant difference between the groups.

\subsection{Stone Free Rates}

The percentage of stone free rates in the present study for supine PCNL was $76 \%$ and for prone PCNL it was $60 \%$ and the difference was statistically significant.

This is in accordance with the RCTs by Jones et al [14] and Sohail et al [15] which are the newer studies using the modified supine positions. But meta-analysis by Yuan D, et al [16] and a systematic review by de la Rosette et al [6] and two multicenter prospective RCT by Valdivia JG et al [17] and by Astroza et al [18] provided contradictory evidence with higher stone rates with prone PCNL than supine PCNL. Other studies which showed a similar success rates between the two procedures were meta-analysis by $\mathrm{Wu} \mathrm{P}$, et al and Liu L, et al $[10,19]$, systematic review by Mak DK et al, Patel RM, et al and Bassiri et al [4, 11, 20].

The reason for the disparity in literature may be due to the use of the original supine position in older studies for supine PCNL which couldn't allow for simultaneous ECIRS and more experience with prone PCNL in comparison to supine PCNL in the older studies.

\subsection{Mean Operating Time}

Mean operating time in present study for patients was calculated from the time of cystoscopy and ureteral catheter placement to percutaneous nephrostomy site closure. It was 101.74 minutes for patients in supine group and 102 minutes in prone group. It was marginally less in supine group but the difference was not statistically significant. This could be because of multiple accesses for stone clearance and increased surgeon experience with prone PCNL.

This is in accordance with the meta-analysis by Yuan D, et al, Wu P, et al, Liu L, et al [10, 16, 19]; systematic reviews by Patel, et al, Bassiri, et al $[4,11]$; prospective RCTs by Jones, et al and Sohail et al $[14,15]$. The explanation was attributed to lack of patient repositioning into prone position, dependent position of Amplatz sheath allowing small fragment to pass spontaneously, less bleeding, improved visibility. It is contradictory to the systematic review by de la Rosette, et al [6] and multicenter RCTs by Valdivia, et al and Astroza, et al $[17,18]$ and prospective RCTs by Wang et al [21] which could be due to the definition of operative time which was calculated from the time of first puncture to the completion of stone removal.

\subsection{Complications/Morbidity}

The complications assessed were fever, bleeding, infection, renal colic. Modified Clavien Dindo grading system was used for comparing the postoperative complications between the two groups. The difference for all the 5 grades between the two groups of supine and prone PCNL was statistically not significant. There was more incidence of fever in the prone group which was statistically significant. The blood transfusion requirements between the two groups were statistically insignificant. The pain VAS scores were more for the prone group which was statistically significant.

This is in accordance with the meta analysis by Yuan D, et al, $\mathrm{Wu} \mathrm{P}$, et al and Liu L, et al [10, 16, 19]; Systematic reviews by Mak DK, et al, Patel RM, et al, de la rosette, et al $[20,4,6]$; Multicenter RCT by Valdivia JG, et al which showed higher incidence of fever for prone group and another by Astroza G, et al. [17, 18] and RCTs by Wang, et al and Sohail, et al $[21,15]$. It is contradictory to the multicenter RCT by Valdivia, et al and single center RCT by Jones $\mathrm{MN}$, et al. [17, 14] which showed a higher complication rate for prone than supine PCNL.

\section{Duration of Hospital Stay}

Average duration of hospital stay was more for prone than supine group but the difference was not statistically significant.

This is in accordance with systematic review by Bassiri A, et al and RCTs by Jones MN, et al and by Sohail N, et al [11, $14,15]$. The studies which show a similar length of hospital stay between two groups include Meta-analysis by Yuan D, et al and by $\mathrm{Wu}$ P, et al $[16,19], \mathrm{RCT}$ by Wang, et al [21].

\section{Requirement of Second Stage PCNL or Other Ancillary Procedures}

Requirement of second stage PCNL or other ancillary procedures were equal in each group.

RCT by Wang, et al and by Sohail, et al showed higher requirement of second stage PCNL or other ancillary procedures for supine group $[21,15]$. This is contrary to the RCT by Sofer M, et al which showed more requirement of additional procedures in prone group [22].

\section{Additional Parameters Studied Which Were Statistically Significant}

The mean stone diameter had a statistically significant difference between the two groups with the prone group having larger size.

The supine group had lesser requirement of nephrostomy tube post procedure which was statistically significant. Nephrostomy tube was not placed if the operating time was less, intraoperative bleeding was less, reduced residual stone burden and no pelvicalyceal system injury. The duration of hospital stay was shorter in the patients who underwent tubeless PCNL compared to those who had a nephrostomy tube at the end of the procedure as per several studies [23]. Multiple RCTs by Al-Dessoukey, et al [24], Sofer M, et al [22], Jones MN, et al [14] have shown that tubeless PCNL are performed more in the supine position than in prone position.

The prone group had a greater requirement of additional punctures for stone clearance which was statistically 
significant. This could be explained by the fact that the prone group had a larger mean stone diameter and there was more space for a second puncture and additional access for stone clearance. The available evidence from RCTs by Wang Y, et al [21], Al-Dessoukey, et al [24], Vincentini FC, et al [25] showed that the difference between the two groups is not statistically significant.

Our study shows that supine PCNL is a better technique than prone PCNL for stone free rates, complication rates, less requirements of nephrostomy tube and lesser number of punctures for stone clearance. This can greatly improve overall patient satisfaction.

There were some limitations of the present study such as Anaesthesiological parameters were not assessed in the study. Stone free rate was kept as less than $4 \mathrm{~mm}$, whereas newer studies require it to be less than $2 \mathrm{~mm}$. The surgery was done by a team of surgeons with varying levels of experience in both supine and prone PCNL. Mean stone diameters for stones was more in the prone group.

\section{Conclusion}

Supine PCNL and ECIRS though in its infancy have added significant benefits to both the patients and the surgeon. This technique is being embraced by Urologists the world over even in complex anatomies of the upper urinary tract. In our study supine PCNL demonstrated better efficacy in terms of stone free rates, lesser post operative morbidity, lesser number of punctures and requirement of nephrostomy tube when compared to Prone PCNL. Supine PCNL as a technique provides the unique opportunity for a surgeon to combine Flexible Ureteroscopy if required (ECIRS) and minimal post operative complications to the patients as compared to prone PCNL. As the technique is embraced by more urologists around the world a true meta analysis comparing Prone and Supine PCNL would be possible. Till such a time surgeon preference and few patient factors like morbid Obesity that would prevent putting the patient prone would determine the choice of PCNL.

\section{References}

[1] Turk C, Skolarikos A, Neisius A et al. EAU guidelines on urolithiasis 2019. [Online]; 2019 [cited 2019 April 30. Available from: https://uroweb.org/individual-guidelines/nononcology-guidelines/urolithiasis.

[2] Assimos D, Krambeck A, Miller NL et al. Surgical Management of Stones: AUA/Endourology Society Guideline (2016). [Online]; 2016 [cited 2019 April 30. Available from: www.auanet.org/guidelines/kidney-stones-surgicalmanagement-guideline.

[3] P, Alken. The Early History of Percutaneous Nephrolithotomy (PNL). In Scoffone CM HACC. Supine Percutaneous Nephrolithotomy and ECIRS: Springer; 2014. p. 5, 6, 7.

[4] Patel RM, Okhunov Z, Clayman RV et al. Prone versus Supine Percutaneous Nephrolithotomy: What is You Position? Current Urology Reports. 2017 April; 18 (4).
[5] Fernstrom I, Johansson B. Percutaneous Nephrolithotomy. A new extraction technique. Scand J Urol Nephrol. 1976; 10 (3).

[6] de la Rosette JJ, Tsakiris P, Ferrandino MN, Elsakka AM, Rioja J, Preminger GM. Beyond prone position in percutaneoous nephrolithotomy: a comprehensive review. Eur Urol. 2008; 54.

[7] Valdivia-Uria JG, Lachares Santamaria E, Villarroya Rodriguez S, et al. Percutaneous nephrolithectomy: simplified technic. Arch Esp Urol. 1987; 40.

[8] Kamphuis GM, Baard J, Westendarp M, de la Rosette JJ. Lessons learned from the CROES percutaneous nephrolithotomy global study. World J Urol. 2015; 33.

[9] Ibarluzea G, Scoffone CM, Cracco CM, Poggio M, Porpiglia F, Terrone C, et al. Supine Valdivia and modified lithotomy position for simultaneous anterograde and retrograde endourological access. BJU Int. 2007; 100.

[10] Liu L, Zheng Shuo, et al. Systematic Review and Metaanalysis of Percutaneous Nephrolithotomy for patients in the supine versus prone position. Journal of Endourol. 2010 December; 24 (12).

[11] Abbas Basiri, Mehrdad Mohammadi Sichani. Supine Percutaneous Nephrolithotomy. Is it really effective? Urol J. 2009; 6 (2).

[12] Falahatkar S, Allahkhah A, Soltanipour S. Supine Percutaneous Nephrolithotomy. Urol J. 2011 May; 8 (4).

[13] Thomas K, Smith NC, Hegarty N, Glass JM. The Guys Stone score - grading the complexity of percutaneous nephrolithotomy procedures. Urology. 2011; 78.

[14] Jones MN, Ranasinghe W, et al. Modified supine versus prone percutaneous nephrolithotomy: Surgical outcomes from a tertiary teaching hospital. Invest Clin Urol. 2016 July; 57 (4).

[15] Sohail N, et al. Percutaneous Nephrolithotomy in complete supine flank-free position in comparison to prone position: A single-centre experience. Arab J Urol. 2017 March; 15 (1).

[16] Yuan D, et al. Supine versus Prone position in percutaneous nephrolithotomy for Kidney Calculi: A Meta-analysis. J Endourol. 2016 July; 30 (7).

[17] Valdivia JG, Scarpa RM, Duvdevani, et al. Supine versus prone position during percutaneous nephrolithootomy: A report from the clinical research office of the endourological society percutaneous nephrolithotomy global study. J Endourol. 2011 October; 25 (10).

[18] Astroza G, Lipkin M, Neisius A, De Sio M, et al. Effect of supine vs prone position on outcomes of percutaneous nephrolithotomy in staghorn calculi: Results from the clinical research office of the endourology society study. Urology. 2013 December; 82 (6).

[19] Wu P, Wang Li, Wang K. Supine versus prone position in percutaneous nephrolithotomy for kidney calculi: a metaanalysis. Int Urol Nephrol. 2011; 43.

[20] Mak DK, Smith Y, et al. What is better in percutaneous nephrolithotomy - Prone or supine? A systematic review. Arab J Urol. 2016 March; 14 (2).

[21] Wang Y, et al. Prone versus modified supine position in percutaneous nephrolithotomy: a prospective randomized study. Int J Med Sci. 2013 September; 10 (11). 
[22] Sofer M, Tavdi E, et al. Implementation of supine percutaneous nephrolithotomy: a novel position for an old operation. Cent Europen J Urol. 2017; 70.

[23] Shen P, Liu Y, Wang J. Nephrostomy tube-free versus nephrostomy tube for renal drainage after percutaneous nephrolithotomy: a systematic review and meta-analysis. Urol Int. 2012; 88 .
[24] Al-Dessoukey AA, Salah Moussa A, Abdelbary A, et al. Percutaneous Nephrolithotomy in the oblique supine lithotomy position and prone position: A comparative study. J Endourol. 2014 September; 28 (9).

[25] Vincenti FC, Perrella R, Souza MG, et al. Impact of patient position on the outcomes of percutaneous nephrolithotomy for complex kidney stones. Int Braz J Urol. 2018 July; 44. 